\title{
A Regularized Visibility-Based Approach to Astronomical Imaging Spectroscopy*
}

\author{
M. Prato ${ }^{\dagger}$, M. Piana ${ }^{\ddagger}$, A. G. Emslie ${ }^{\S}$, G. J. Hurford , E. P. Kontarll, and A. M. Massone\#
}

Abstract. We develop a formal procedure for the analysis of imaging spectroscopy data, i.e., remote sensing observations of the structure of a radiation source as a function of an observed parameter (e.g., radiation wavelength, frequency, or energy) and two-dimensional location in the observation plane of the instrument used. In general, imaging spectroscopy involves inversions of both spatial and spectral information. "Traditional" approaches typically proceed by performing the spatial inversion first, and then applying spectral deconvolution algorithms on a "pixel-by-pixel" basis across the source to deduce the (line-of-sight-weighted) form of the "source function" (a function involving only physical properties of the source itself) at each location in the observation plane. However, in the special case where spatial information is encoded in the form of visibilities (two-dimensional spatial Fourier transforms of the source structure), it is advantageous, both conceptually and computationally, to reverse the order of the steps in this procedure. In such an alternative approach, the spectral inversion is performed first, yielding visibilities of the unknown source function, and then these source function visibilities are spatially transformed to yield in situ information on the source, as a function of both energy and position. We illustrate the power and fidelity of this method using simulated data and apply it to hard X-ray observations of a solar flare on April 15, 2002. We also discuss briefly its broader applicability.

Key words. astronomical imaging, Fourier transforms, remote sensing, solar flares, spectroscopy, spectral inversion, visibilities, X-rays

AMS subject classifications. 65R32, 68U10, 85-08

DOI. $10.1137 / 090746355$

\footnotetext{
${ }^{*}$ Received by the editors January 12, 2009; accepted for publication (in revised form) July 6, 2009; published electronically September 9, 2009. This work was supported by a grant from the International Space Science Institute (ISSI) in Bern, Switzerland.

http://www.siam.org/journals/siims/2-3/74635.html

${ }^{\dagger}$ Dipartimento di Matematica Pura e Applicata, Università di Modena e Reggio Emilia, via Campi 213/b, 41100 Modena, Italy, and CNR-INFM LAMIA, via Dodecaneso 33, 16146 Genova, Italy (marco.prato@unimore.it). This author's research was supported by contract I/015/07/0 between the Italian ASI, the Italian INAF, and the Italian GNCS.

‡Dipartimento di Informatica, Università di Verona, Strada le Grazie 15, Ca' Vignal 2, 37134 Verona, Italy, and CNR-INFM LAMIA, via Dodecaneso 33, 16146 Genova, Italy (michele.piana@univr.it). This author's research was supported by contract I/015/07/0 between the Italian ASI and the Italian INAF.

${ }^{\S}$ Department of Physics, Oklahoma State University, Stillwater, OK 74078 (gordon.emslie@okstate.edu). This author's research was supported by NASA grant NNG04G063G and by subcontract SA4878-26308 from the University of California, Berkeley.

ISpace Sciences Laboratory, University of California at Berkeley, 7 Gauss Way, Berkeley, CA 94720 (ghurford@ ssl.berkeley.edu). This author's research was supported by NASA contract NAS5-98033.

"Department of Physics and Astronomy, University of Glasgow, Glasgow G12 8QQ, Scotland, UK (eduard@astro. gla.ac.uk). This author's research was supported by a PPARC Advanced Fellowship.

\#CNR-INFM LAMIA, via Dodecaneso 33, 16146 Genova, Italy (massone@ge.infm.it). This author's research was supported by contract I/015/07/0 between the Italian ASI and the Italian INAF.
}

910

Copyright (C) by SIAM. Unauthorized reproduction of this article is prohibited. 
1. Introduction. As with all remote sensing investigations, astronomical observations routinely pose the challenge of how to deduce, from observable properties of a radiation field (e.g., the spectrum, plane-of-the-sky image, and/or polarization of the photon flux produced by a distant star or nebula), information on some physically significant property (e.g., temperature, density) of the source itself. In particular, imaging spectroscopy generally involves determining, from a series of observed images at different values of an observational parameter (e.g., wavelength, frequency, energy), two-dimensional maps of a source function of interest (e.g., mean particle distribution) at different values of an associated source variable (e.g., particle energy).

From a mathematical viewpoint, imaging spectroscopy involves the solution of two inverse problems, both of which are generally ill-posed. First, spatial information is degradated (e.g., distorted, blurred) by the instrumental response of the imaging device, typically expressed in terms of the point spread function (PSF). Second, the observed spectrum is related to the source function through the spectral relationship between the observed parameter and the source function variable. Both these problems can be represented mathematically by linear integral equations of the first kind: for imaging models, the kernel of the pertinent integral equation is the PSF; for spectral inversion, the kernel represents the probability that emission at a particular value of the observational parameter occurs as a result of a process involving a particular value of the source function variable.

Astronomical imaging spectroscopy using inverse methods is typically accomplished by first applying regularization methods to the problem of reconstructing two-dimensional images through spatial inversion procedures, and then to the problem of performing a spectral inversion from the observed spectrum to the source function distribution. Figure 1 shows a pictorial description of such a natural methodology. In such a scheme we suppose that a $K_{1}$-pixel $\times K_{2}$-pixel (blurred) image of the emission from the source is available at each of $N$ different values of an observational parameter (e.g., radiated frequency). Then the following occur in order:

1. Each (blurred) image is processed by means of a spatial inversion method to obtain a reconstructed image in which the artifacts related to the PSF of the imaging device are reduced. The result is a set of $N$ reconstructed $K_{1} \times K_{2}$ images of the emission from the source, one for each of the $N$ different values of the observational parameter.

2. For each of the $K_{1} K_{2}$ pixels in the image, a one-dimensional $N$-plot is constructed of the pixel content versus the observational parameter. This procedure is repeated for all pixels to obtain $K_{1} K_{2} N$-plots of the observed spectrum.

3. Using a regularization method, the observed spectrum corresponding to each pixel is spectrally inverted to obtain the corresponding regularized source spectrum (i.e., a onedimensional plot of the source function versus the source variable) for that particular pixel. This procedure is repeated for all pixels to obtain $K_{1} K_{2}$ regularized source spectra. Note that in general regularized inversion methods work for "rectangular" problems (e.g., [9]), so that we can obtain values for the source function for $M \geq N$ values of the source variable.

4. From the $K_{1} K_{2}$ source spectra thus obtained, we take, for a specified value of the source variable, the $K_{1} K_{2}$ values of the regularized source function in each pixel within the image. Repeating this for each of the $M$ values of the source variable results in a set

Copyright (c) by SIAM. Unauthorized reproduction of this article is prohibited. 


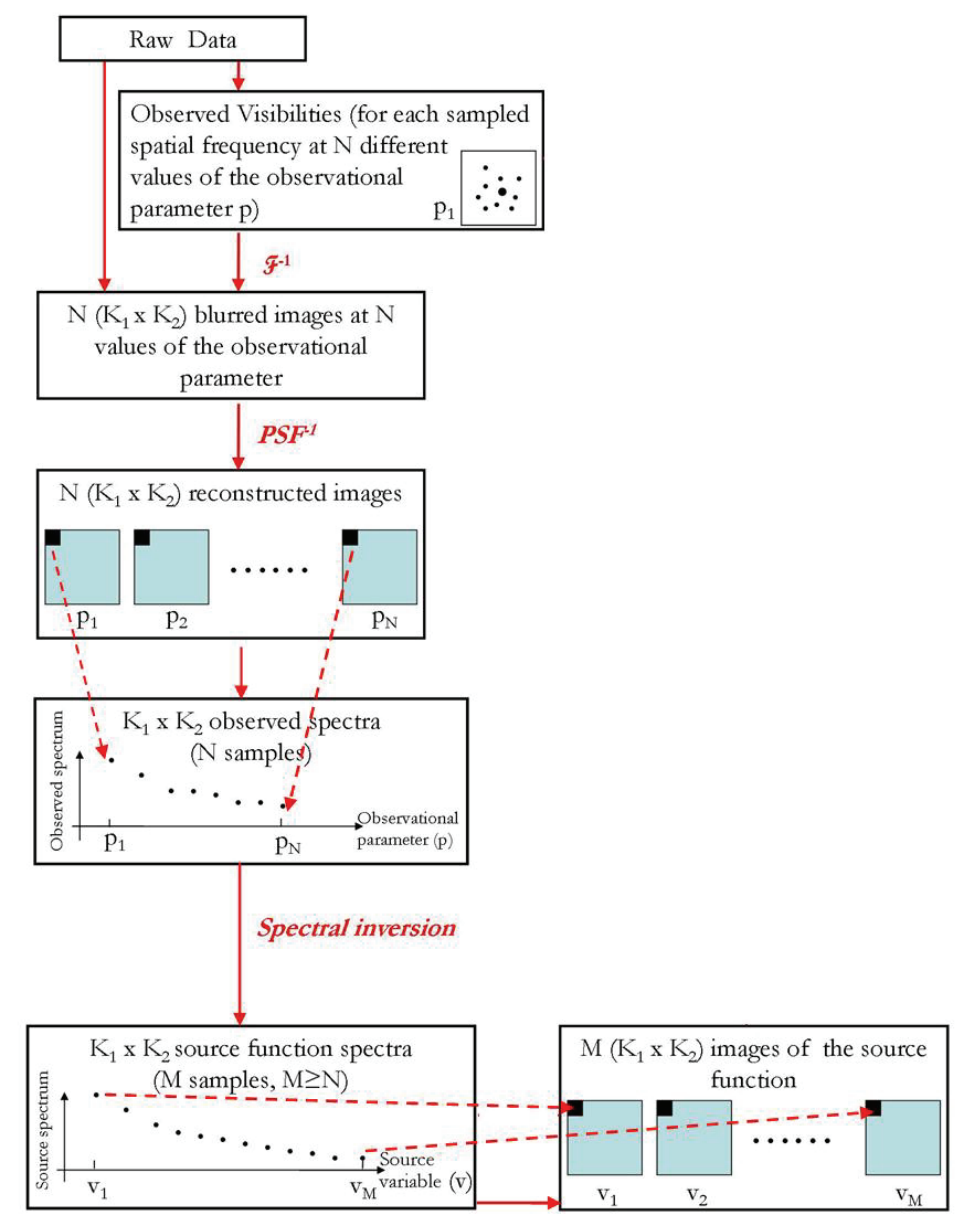

Figure 1. Traditional imaging spectroscopy approach. The raw data may be either in the form of pixelby-pixel ("rastered") images or in the form of Fourier components ("visibilities") which are spatially inverted $\left(\mathcal{F}^{-1}\right)$ to obtain images. The observed images are first subjected to a spatial deconvolution (denoted by $P S F^{-1}$ ). Then, the observed spectrum for the same pixel in all images is inverted, resulting in source spectra for each pixel. Rearrangement of this information results in images of the source function at each value of the source variable.

of $K_{1} \times K_{2}$ regularized images of the source function, one for each of the $M$ different values of the source variable.

This approach is characterized by two very significant drawbacks. First, the overall computational cost of the method is considerable; step 3 alone requires the regularized inversion of $K_{1} K_{2}$ (typically $\sim 10^{4}-10^{6}$ ) observed spectra. Second, the input data for these spectral inversions are the pixel contents of the images reconstructed in step 1; these input values are the result of a previous ill-posed (spatial) inversion, which has weakened their information content.

For certain types of observation, however, there is an approach which effectively overcomes both of these drawbacks. For such observations, imaging information is obtained not on 
a "pixel-by-pixel" basis, but rather through calibrated measurements of specific spatial Fourier components of the emitted radiation field. Examples include multielement interferometry (at radio, infrared, and optical wavelengths) [19], X-ray imaging using spatially modulating (e.g., Moiré fringe) techniques [13], and X-ray imaging using temporal modulation. An example of the last technique involves rotating modulation collimators (RMCs), in which spatial information is encoded in rapid time variations of the detected counts [7]. In all cases, the measured values of the spatial Fourier transform of the radiation flux are termed visibilities. Visibilities represent readily processable data in this kind of astronomy, since

- they are fully calibrated, containing no instrumental dependence other than instrumentally defined spatial frequencies;

- their statistical error is well-defined, since they are formed by a straightforward linear combination of detected intensities/counts;

- in general, since noise and background do not possess a characteristic spatial/temporal frequency, they do not bias the observed visibilities;

- in certain cases (e.g., RMCs), the symmetry properties of the imaging system offer a level of redundant information and so provide an indication of systematic errors.

Finally, we note that visibilities encode the spatial information content in a very efficient way, particularly in sparse images (i.e., those in which only a minority of the pixels have a nonzero content), since it is possible to infer information on the essential spatial properties of a source from a rather limited set of visibilities.

The possibility of the raw data being in visibility form is included in Figure 1; in such a case an inverse Fourier transform $\left(\mathcal{F}^{-1}\right)$ is performed to yield images for each value of the observational parameter $p$. There is, however, an alternative way of proceeding, introduced by Piana et al. [14] in the context of studying solar flares with observations from the RMC-based instrument on the NASA Reuven Ramaty High-Energy Solar Spectroscopic Imager (RHESSI) mission. The approach is founded on the fact that, under rather general assumptions (to be discussed below), the spatial Fourier transforms of the observed and source spectra are related by the same integral equation that relates the observed and source spectra themselves. It is therefore possible to reverse the order of spatial and spectral inversions: the spectral inversion is performed directly on the visibility data, and the resulting source visibilities are inverted spatially to yield source function images. Specifically, in the first step of this twostep scheme, the ill-posed spectral inversion is performed in the spatial frequency domain, employing a regularization technique which utilizes visibilities as input data. This leads to sets of (regularized) source visibilities with each set corresponding to a given value of the source variable. Then, in the second step, maps of the source function at specified values of the source variable are constructed using a Fourier-based image restoration algorithm using as input data the regularized source visibilities at the specified value of the source variable.

This provides a very effective approach to imaging spectroscopy: visibilities represent a reliable data format for the ill-posed spectral inversion, and a relatively small number $L$ of (complex) visibilities at a particular value of the source variable are sufficient to produce useful images at that value. (The number of visibility components, $2 L$, is typically $\ll K_{1} K_{2}$, the number of image pixels.)

The present paper has three essential aims. First, we will provide a formal mathematical formulation of this visibility-based imaging spectroscopy approach in appropriate functional

Copyright (c) by SIAM. Unauthorized reproduction of this article is prohibited. 
spaces. This formulation will allow us to show that this method, originally introduced for RHESSI visibilities, can be generalized to other cases where the "native" form of the data is in the form of visibilities. Second, we will discuss the implementation of the method in the framework of the Solar SoftWare (SSW) tree of the RHESSI mission. Third, we will provide a systematic validation of the method in the case of both synthetic and real data.

The plan of the paper is as follows. In section 2 we describe an example (in solar hard $\mathrm{X}$-ray astronomy) where imaging spectroscopy with visibilities is effective. Section 3 introduces the new reconstruction method in a more general setting, and section 4 describes the implementation of the method in practice. In section 5 a validation of the approach is performed using synthetic data. In section 6 we apply the method to real solar hard X-ray imaging spectroscopy data, and in section 7 we present our conclusions.

2. Solar X-ray bremsstrahlung and the RHESSI mission. The physical process that relates hard X-ray emission to the parent distribution of energetic electrons in solar flare plasmas is optically thin electron-ion bremsstrahlung [3]. In the terminology of the previous section, the observational parameter is the energy $\epsilon$ of the emitted photon; the observed values are the photon flux per unit (two-dimensional) area of the image on the plane of the sky; and the sought-after source function is the spectrum of the energetic electrons, as a function of both position within the source and the source variable $E$, the electron energy.

Let $\mathbf{x}$ be a point in the image plane containing the source, and let $z$ be the distance along the line of sight into the source at point $\mathbf{x}$. Since the source is optically thin, the relation between the photon spectrum $g(\mathbf{x} ; \epsilon)$ (photons $\mathrm{cm}^{-2} \mathrm{~s}^{-1} \mathrm{keV}^{-1} \operatorname{arcsec}^{-2}$ ) emitted at energy $\epsilon(\mathrm{keV})$ from the point $\mathbf{x}$, and the differential electron flux spectrum $F(\mathbf{x}, z ; E)$ (electrons $\mathrm{cm}^{-2} \mathrm{~s}^{-1} \mathrm{keV}^{-1}$ ) at the point $(\mathbf{x}, z)$ and at electron energy $E(\mathrm{keV})$ is given by

$$
g(\mathbf{x} ; \epsilon)=\frac{a^{2}}{4 \pi R^{2}} \int_{\epsilon}^{\infty} \int_{0}^{\ell(\mathbf{x})} n(\mathbf{x}, z) F(\mathbf{x}, z ; E) Q(\epsilon, E) d z d E,
$$

where $R=1 \mathrm{AU}\left(=1.5 \times 10^{13} \mathrm{~cm}\right), a \equiv 7.25 \times 10^{7} \mathrm{~cm} \mathrm{arcsec}^{-1}$ is the conversion factor from angle subtended at the Earth to distance on the solar surface (so that $R / a=206265$ arcseconds (= 1 radian $)$ ), $\ell(\mathbf{x})$ is the line-of-sight depth; $n(\mathbf{x}, z)\left(\mathrm{cm}^{-3}\right)$ is the local plasma density; and $Q(\epsilon, E)\left(\mathrm{cm}^{2} \mathrm{keV}^{-1}\right)$ is the bremsstrahlung cross-section ${ }^{1}$ differential in photon energy $\epsilon$, representing the probability that a photon of energy $\epsilon$ is emitted by an electron of energy $E$. $Q(\epsilon, E)$ vanishes for $\epsilon>E$; i.e., in the bremsstrahlung process, only photons with energy less than or equal to the energy of the emitting electron can be produced. In the allowable range $\epsilon<E, Q(\epsilon, E)$ is a gradually decreasing function of both $\epsilon$ and $E$ (the cross-section for emission of the photon of energy $\epsilon$ decreases as the energy of the emitting electron increases, and a given electron is more likely to produce a low-energy photon than one with energy comparable to the electron itself). The variation of $Q(\epsilon, E)$ with both $\epsilon$ and $E$ is relatively gradual, a factor that contributes to the ill-posedness of the bremsstrahlung equation (2.1) [12]. Such an equation can be simplified by introducing the column density

\footnotetext{
${ }^{1}$ Here we take $Q(\epsilon, E)$ to be independent of the direction of the emitted photon; i.e., we use the isotropic (solid-angle-averaged) form. There is no inherent difficulty in generalizing the results of this paper to anisotropic forms [11] of $Q(\epsilon, E)$.
}

Copyright ( by SIAM. Unauthorized reproduction of this article is prohibited. 
$N(\mathbf{x})\left(\mathrm{cm}^{-2}\right)$ at each point $\mathbf{x}$,

$$
N(\mathbf{x})=\int_{0}^{\ell(\mathbf{x})} n(\mathbf{x}, z) d z
$$

and the definition of the mean electron flux spectrum [5],

$$
\bar{F}(\mathbf{x} ; E)=\frac{1}{N(\mathbf{x})} \int_{0}^{\ell(\mathbf{x})} n(\mathbf{x}, z) F(\mathbf{x}, z ; E) d z .
$$

Using (2.3), (2.1) may be written as

$$
g(\mathbf{x} ; \epsilon)=\frac{1}{4 \pi R^{2}} \int_{\epsilon}^{\infty}\left[a^{2} N(\mathbf{x}) \bar{F}(\mathbf{x} ; E)\right] Q(\epsilon, E) d E .
$$

This can be further simplified by defining

$$
f(\mathbf{x} ; E):=\frac{1}{4 \pi R^{2}}\left[a^{2} N(\mathbf{x}) \bar{F}(\mathbf{x} ; E)\right]
$$

and

$$
K(\epsilon, E)= \begin{cases}Q(\epsilon, E), & E \geq \epsilon \\ 0, & E<\epsilon\end{cases}
$$

so that

$$
g(\mathbf{x} ; \epsilon)=\int_{0}^{\infty} f(\mathbf{x} ; E) K(\epsilon, E) d E .
$$

In (2.4)-(2.7), $f(\mathbf{x} ; E)$ is the source function at different values of the source variable $E$, $g(\mathbf{x} ; \epsilon)$ is the observed image at different values of the observational parameter $\epsilon$, and $Q(\epsilon, E)$ quantifies the physical process relating the source function (mean electron flux spectrum) and observed spectrum (photon spectrum). Analytical formulas for $Q(\epsilon, E)$ are available $[6,8,12]$, accounting for all pertinent physics, including relativistic effects, Coulomb screening, and electron-electron emission.

The scientific goals of the NASA RHESSI mission [10], launched on February 5, 2002, are to study the processes of particle (electron and ion) acceleration and propagation in solar flares. A key element of such investigations is the determination of the accelerated electron spectrum from hard X-ray imaging spectroscopy data, obtained through the solution of (2.7). RHESSI obtains imaging information using a set of nine RMCs, each consisting of a pair of coaligned grids with different pitches. The photon flux transmitted through each RMC is temporally modulated by the rotating grids and is recorded on a set of cooled Ge detectors, which provide a spectral resolution of around $1 \mathrm{keV}$ over a spectral range of around 5-500 keV. Imaging information is encoded in the observed temporal modulation pattern; at a given instant the amplitude and phase of this modulation pattern directly measure the amplitude and phase of a particular two-dimensional spatial Fourier component. These Fourier components, termed visibilities, are measured at spatial frequencies $(u, v)$ corresponding to the angular resolutions of the various RMCs. 


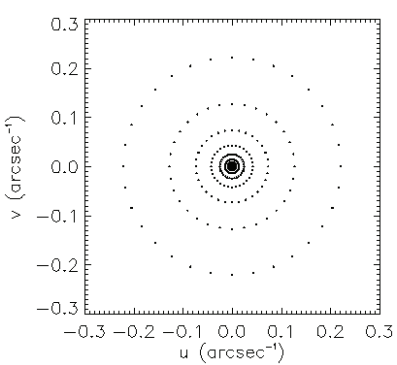

(a)

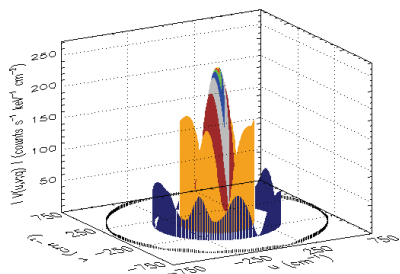

(b)

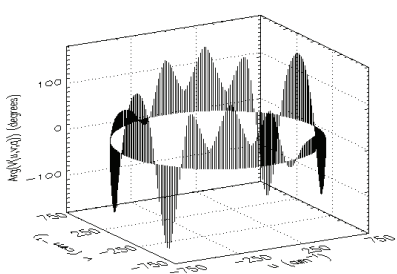

(c)

Figure 2. Sampling (a) of the spatial frequency $(u, v)$ plane by the nine RMC subcollimators on RHESSI, together with an illustration of the (smoothed) amplitude (b) and phase (c) of the visibilities in a particular photon energy bin for a simulated event. For pictorial reasons, in (b) we have reported the visibilities associated to seven detectors over nine and in (c) to one detector over nine.

Each RMC measures Fourier components on a circle of constant radius $\sqrt{u^{2}+v^{2}}$ in the spatial frequency domain. In order to produce a set of independent Fourier components, this circle is sampled at a finite number of $(u, v)$ points lying on this circle. An example sampling in the $(u, v)$-plane is shown in the left panel of Figure 2: each circle corresponds to a single detector, and each dot denotes a sampled $(u, v)$ frequency at which a visibility (a complex number) is measured, in a set of (user-prescribed) photon energy bins. Pictorial views of the amplitude and phase of some typical visibilities, for some subset of the nine detectors at a specific photon energy $\epsilon$, are given in the middle and right panels of Figure 2, respectively. In the imaging spectroscopy approach discussed in the present paper, the sets of all visibilities provided by all the available collimators, in all photon energy bins, are the input data from which we wish to infer maps of the source function $f(\mathbf{x} ; E)$.

3. Formulation of the method. The aim of the present section is to introduce a general visibility-based imaging spectroscopy procedure for the reconstruction of spatial maps $f(\cdot ; E)$ of the source function, at specified values of the source variable $E$.

Within a functional analysis framework it is advantageous to introduce the operator $A$ : $L^{1}\left(\mathbb{R}^{2}, L^{2}(0, \infty)\right) \rightarrow L^{1}\left(\mathbb{R}^{2}, L^{2}(0, \infty)\right)$ such that $A f$ is the map defined by

$$
A f: \mathbf{x} \rightarrow \int_{0}^{\infty} K(\cdot, E) f(\mathbf{x} ; E) d E,
$$

with $\mathbf{x} \in \mathbb{R}^{2}$. The space $L^{1}\left(\mathbb{R}^{2}, L^{2}(0, \infty)\right)$ is the Banach space of all functions $f$ defined on $\mathbb{R}^{2}$ with values in $L^{2}(0, \infty)$, equipped with the Böchner norm

$$
\|f\|=\sqrt{\int_{\mathbb{R}^{2}}\left(\int_{0}^{\infty}|f(\mathbf{x} ; E)|^{2} d E\right) d \mathbf{x} .}
$$

In this framework, definition $(3.1)$ is well-posed, since if $K(\cdot, \cdot)$ is a Hilbert-Schmidt kernel, then $A$ is a compact operator. For the sake of clarity, from now on we denote with $\hat{\mathbb{R}}^{2}$ the frequency domain and with $\hat{A}$ the operator $A$ when it acts on a function parameterized over 
$\hat{\mathbb{R}}^{2}$; i.e., $\hat{A}: L^{1}\left(\hat{\mathbb{R}}^{2}, L^{2}(0, \infty)\right) \rightarrow L^{1}\left(\hat{\mathbb{R}}^{2}, L^{2}(0, \infty)\right)$ is such that

$$
\hat{A} f: \boldsymbol{\omega} \rightarrow \int_{0}^{\infty} K(\cdot, E) f(\boldsymbol{\omega} ; E) d E,
$$

with $\boldsymbol{\omega} \in \hat{\mathbb{R}}^{2}$. We then use the Fourier transform of a function in $L^{1}\left(\mathbb{R}^{2}, L^{2}(0, \infty)\right)$, defined as the map $\mathcal{F}: L^{1}\left(\mathbb{R}^{2}, L^{2}(0, \infty)\right) \rightarrow L^{1}\left(\hat{\mathbb{R}}^{2}, L^{2}(0, \infty)\right)$ such that

$$
\mathcal{F} f: \boldsymbol{\omega} \rightarrow \int_{\mathbb{R}^{2}} e^{-i \boldsymbol{\omega} \cdot \mathbf{x}} f(\mathbf{x} ; \cdot) d \mathbf{x}
$$

The result at the basis of our new approach to imaging spectroscopy is that, again for HilbertSchmidt kernels, Fubini's theorem straightforwardly implies the commutative property

$$
\mathcal{F} A=\hat{A} \mathcal{F} \text {. }
$$

Equation (3.5) then allows us to write an imaging spectroscopy equation in the frequency domain, namely,

$$
\hat{g}(\boldsymbol{\omega} ; \epsilon)=\int_{0}^{\infty} K(\epsilon, E) \hat{f}(\boldsymbol{\omega} ; E) d E,
$$

where $\hat{f} \equiv \mathcal{F} f ; \hat{g} \equiv \mathcal{F} g$. Equation (3.6) is the continuous representation of an observation process in which visibilities represent the most native measurements. In the present setting, the measured visibilities are represented by

$$
V_{i}\left(\epsilon_{k}\right)=\hat{g}\left(\boldsymbol{\omega}_{i} ; \epsilon_{k}\right)+\delta_{i k},
$$

where the (complex) visibilities $V_{i}\left(\epsilon_{k}\right)$ are the experimental values of the Fourier transform of $g\left(\mathbf{x} ; \epsilon_{k}\right)$ at the value $\epsilon_{k}$ of the observational parameter $\epsilon$ and at the point $\boldsymbol{\omega}_{i}=\left(u_{i}, v_{i}\right)$ in the frequency $(u, v)$-plane, and $\delta_{i k}$ is the corresponding observation error. In analogy with these experimental visibilities we introduce the visibilities associated with the source function $f(\mathbf{x} ; E)$; specifically, we denote with $W_{i}\left(E_{j}\right)$ a complex number which is the value of the Fourier transform of $f\left(\mathbf{x} ; E_{j}\right)$ at the value $E_{j}$ of the source variable $E$, also at the point $\left(u_{i}, v_{i}\right)$ in the frequency $(u, v)$-plane; i.e.,

$$
W_{i}\left(E_{j}\right)=\hat{f}\left(\boldsymbol{\omega}_{i} ; E_{j}\right) .
$$

$V_{i}\left(\epsilon_{k}\right)$ and $W_{i}\left(E_{j}\right)$ are related by the discretized version of (3.6):

$$
V_{i}\left(\epsilon_{k}\right)=\sum_{j=1}^{M} K\left(\epsilon_{k}, E_{j}\right) W_{i}\left(E_{j}\right) \delta E_{j}, \quad k=1, \ldots, N, \quad i=1, \ldots, L,
$$

where $\delta E_{j}$ refers to the quadrature form adopted. Owing to the compactness of $A$, the linear systems (3.9) are ill-conditioned.

On the basis of (3.9), a new imaging spectroscopy algorithm, described in Figure 3, can be formulated. According to this scheme, we do the following:

Copyright (C) by SIAM. Unauthorized reproduction of this article is prohibited. 


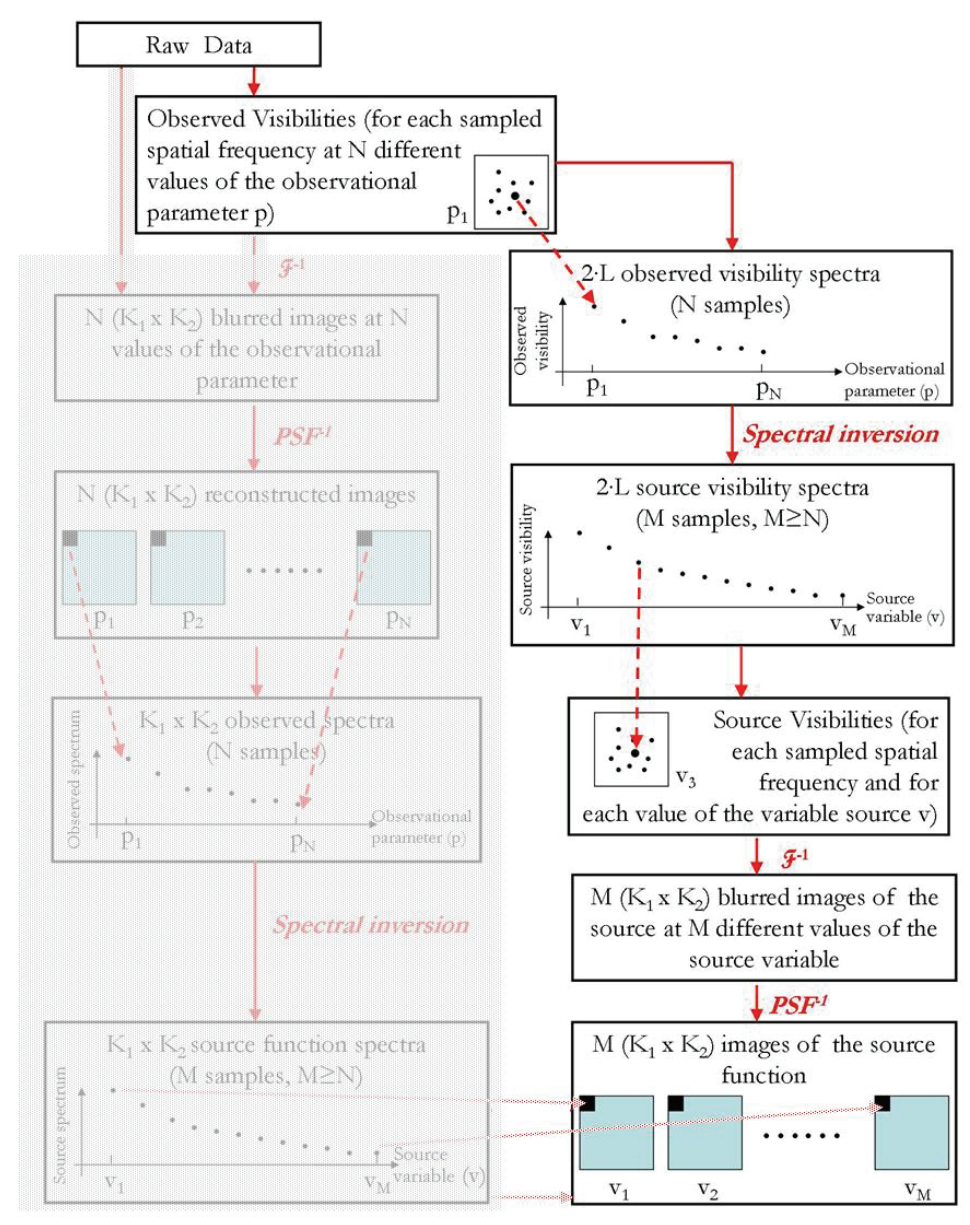

Figure 3. New (visibility-based) imaging spectroscopy method. Note that both the traditional method (Figure 1) and the new method produce images of the source function through application of three processes: spatial inversion $\left(P S F^{-1}\right)$, spectral inversion, and inverse spatial Fourier transform $\left(\mathcal{F}^{-1}\right)$. However, in the new method the order of application of these steps is optimized for the type of raw data available; it proceeds first with a spectral inversion in the spatial frequency domain, then with a Fourier inversion to obtain (blurred) source images, and finally with a spatial deconvolution to remove the PSF. For application to the solar hard $X$-ray bremsstrahlung problem, we make the identifications $p \equiv \epsilon$ (photon energy) and $v \equiv E$ (electron energy).

1. We denote with

$$
\mathcal{V}=\left\{V_{i}\left(\epsilon_{k}\right), \quad k=1, \ldots, N, \quad i=1, \ldots, L\right\}
$$

the set of all measured visibilities for all sampled values of the parameter $\epsilon$; for a fixed value of $i, V_{i}\left(\epsilon_{k}\right), k=1, \ldots, N$, represents a measured (complex) visibility spectrum as a (discretized) function of the parameter $\epsilon$.

2. A regularization method is applied to the solution of (3.9) to obtain a reconstructed (complex) visibility spectrum $W_{i}^{\boldsymbol{\lambda}_{i}}\left(E_{j}\right), j=1, \ldots, M$, where $\boldsymbol{\lambda}_{i}$ is a 2 -vector, specific to each visibility $i$, that gives the regularization parameters used in the inversion of

Copyright (c) by SIAM. Unauthorized reproduction of this article is prohibited. 
the real and imaginary components of that visibility spectrum. By repeating this regularized inversion for all sampled frequencies $\left(u_{i}, v_{i}\right), i=1, \ldots, L$, one obtains the set of all reconstructed source visibilities

$$
\mathcal{W}=\left\{W_{i}^{\boldsymbol{\lambda}_{i}}\left(E_{j}\right), \quad j=1, \ldots, M, \quad i=1, \ldots, L\right\} .
$$

3. For a fixed value of $j$, a Fourier inversion imaging method is applied to $W_{i}^{\boldsymbol{\lambda}_{i}}\left(E_{j}\right)$, $i=1, \ldots, L$, to obtain a reconstruction of $f(\mathbf{x} ; E)$ corresponding to the value $E_{j}$ of the source variable $E$. Repeating this reconstruction procedure for all values of $j$ leads to the desired imaging spectroscopy result: a visualization of source function maps $f(\mathbf{x} ; E)$ for many values of the source variable $E$ (electron energy).

This imaging spectroscopy method has four significant advantages:

1. The proposed approach proceeds via a set of electron visibilities $\mathcal{W}(E)$ which are, by virtue of the regularized process used to construct them, smoothly varying with respect to the source variable $E$. Consequently, the resulting images of the source function also vary smoothly with respect to $E$, a very natural physical constraint.

2. In the proposed approach, measurements in their most native form (complete with straightforwardly estimated uncertainty values) are utilized as input data for the most ill-posed (spectral) inverse problem. This contrasts with the traditional approach, in which a nonlinear spatial inversion algorithm (e.g., maximum entropy or clean) is applied first, providing reconstructed pixel values which will be used as input data for the most unstable part of the overall algorithm.

3. The number of regularized spectral inversions that need to be performed can be substantially less than the number required in the traditional pixel-by-pixel approach. Specifically, for each sampled point in the $(u, v)$-plane, one requires only two inversions, one corresponding to the real part and one to the imaginary part of each measured visibility spectrum. The overall number of inversions can be considerably less than the number of pixels in the image.

4. The final image reconstruction step, which can be extremely computationally demanding, is here essentially simply a Fourier transform inversion problem with incomplete data, which can be effectively addressed by applying proven Fourier methods.

4. Implementation of the method. In the present section we describe the main issues concerning the practical implementation of the new imaging spectroscopy method; such an implementation has been incorporated into an IDL code available within the SSW tree (see http://www.lmsal.com/solarsoft/). The issues to be discussed involve the nature of the input data, the regularization procedure adopted for the spectral inversion, and the image reconstruction techniques which can be applied to visualize the source function from the regularized electron visibilities.

4.1. Input data. The statistical quality of photon ${ }^{2}$ visibilities depends on the overall energetics of the observed flare, on the energy of the photon channel involved, and on the

\footnotetext{
${ }^{2}$ Technically, there is a distinction between detected counts and the photons that produce them. The relationship depends on details of the detector response (e.g., [18]) and is not pertinent to the purposes of the present paper. Hereafter, we shall use the term "photons" to denote both actual photons (produced by bremsstrahlung on the sun) and counts (which determine, for example, the statistical accuracy of the visibilities used in the analysis).
}

Copyright (c) by SIAM. Unauthorized reproduction of this article is prohibited. 
angular resolution of the RMC, a quantity directly related to the pitch of the RMC grids.

As a general rule, the following considerations are valid:

- The statistical relative noise decreases with an increase in the overall size (energy content) of the flare.

- Because of the steep photon spectra $\left(\sim \epsilon^{-\gamma}\right.$, with $\left.\gamma \sim 3-5\right)$ typical of solar flares, the quality of the measurements rapidly deteriorates for energies $\epsilon \gtrsim 70-80 \mathrm{keV}$.

- For energies $\epsilon \lesssim 10 \mathrm{keV}$, neither free-bound (recombination) nor bound-bound emissions (spectral lines) are negligible, so that the bremsstrahlung cross-section $Q(\epsilon, E)$ is no longer applicable. This renders our results physically unreliable in this range.

- Detectors with narrower slits correspond to finer angular resolution and so generate visibilities at points in the $(u, v)$-plane corresponding to circles with larger radii. These detectors thus provide more statistically significant independent visibilities than detectors with wider slits, corresponding to circles with smaller radii in the $(u, v)$-plane. For a large flare, detectors from 1 to 5 (those with the smaller grid pitches) can typically provide some $30-32$ statistically significant visibilities at energy channels from 10-40 keV; this number decreases to 15-30 visibilities for detectors 6 and 7 and to only $6-15$ visibilities for detectors 8 and 9 .

In the tests of the next section we will use a routine available in SSW to produce synthetic visibilities and then introduce a realistic level of noise characterized by Poisson statistics plus a systematic error which is deduced from known properties of the RHESSI hardware.

4.2. Spectral inversion. We performed the inversion of the photon visibility spectra by applying Tikhonov regularization [1]. The most significant challenge in performing this inversion is the large dynamic range of the pertinent input data: for most frequency pairs $\left(u_{i}, v_{i}\right), i=1, \ldots, L$, the visibility spectra $V_{i}\left(\epsilon_{k}\right), k=1, \ldots, N$, vary by around five orders of magnitude over the useful energy range of 10-70 keV. This rapid decrease of $V_{i}\left(\epsilon_{k}\right)$ with $\epsilon_{k}$ makes the application of Tikhonov regularization problematic, since the required regularization parameter $\boldsymbol{\lambda}_{i}$ is a global parameter, applicable to the entire range of the input data. To accommodate the high dynamic range in the input data we therefore perform a rescaling [9] of (3.6) to the form

$$
\hat{g}^{\prime}(\boldsymbol{\omega} ; \epsilon)=\int_{0}^{\infty} h(E) K^{\prime}(\epsilon, E) \hat{f}(\boldsymbol{\omega} ; E) d E,
$$

with

$$
\begin{aligned}
\hat{g}^{\prime}(\boldsymbol{\omega} ; \epsilon) & :=h(\epsilon) \hat{g}(\boldsymbol{\omega} ; \epsilon), \\
K^{\prime}(\epsilon, E) & :=\frac{h(\epsilon)}{h(E)} K(\epsilon, E),
\end{aligned}
$$

and $h(\cdot)$ an appropriately chosen function. In particular, we considered three possible forms of $h(\epsilon)$ :

$$
\begin{gathered}
h(\epsilon)=A \epsilon^{p} ; \\
h(\epsilon)=A \epsilon^{p}+B \epsilon^{q} ;
\end{gathered}
$$

Copyright (c) by SIAM. Unauthorized reproduction of this article is prohibited. 


$$
h(\epsilon)= \begin{cases}A \epsilon^{p}, & \epsilon \leq \epsilon_{0} \\ B \epsilon^{q}, & \epsilon>\epsilon_{0}\end{cases}
$$

The measured visibility spectrum $V_{i}\left(\epsilon_{k}\right), k=1, \ldots, N$, at each applicable $\left(u_{i}, v_{i}\right)$ is fitted by means of the three forms (4.4)-(4.6) and, for each visibility $i$, the form that provides the smallest $\chi^{2}$ value, and the corresponding values of $p, q$, and $\epsilon_{0}$ (as appropriate), are determined. After this rescaling, the actual spectroscopic problem to be numerically solved by our method becomes the set

$$
h\left(\epsilon_{k}\right) V_{i}\left(\epsilon_{k}\right)=\sum_{j=1}^{M} h\left(E_{j}\right) K^{\prime}\left(\epsilon_{k}, E_{j}\right) W_{i}\left(E_{j}\right) \delta E_{j}, \quad k=1, \ldots, N, \quad i=1, \ldots, L .
$$

We solve these by applying the Tikhonov method at each sampled $\left(u_{i}, v_{i}\right)$ point, $i=1, \ldots, L$; i.e., we solve the minimization problem

$$
\left\|\mathbf{A} \operatorname{Re}\left(\mathbf{W}_{i}\right)-\operatorname{Re}\left(\mathbf{G}_{i}\right)\right\|^{2}+\lambda_{i}\left\|\operatorname{Re}\left(\mathbf{W}_{i}\right)\right\|^{2}=\operatorname{minimum}
$$

and a similar equation for the imaginary parts. Here $\|\cdot\|$ is the Euclidean norm, A (as determined for each visibility $i$ ) is the $N \times M$ matrix with entries

$$
\mathbf{A}_{k j}=h\left(E_{j}\right) K^{\prime}\left(\epsilon_{k}, E_{j}\right) \delta E_{j}, \quad k=1, \ldots, N, \quad j=1, \ldots, M,
$$

$\mathbf{W}_{i}$ is the $M$-vector with components

$$
\left(\mathbf{W}_{i}\right)_{j}=W_{i}\left(E_{j}\right), \quad j=1, \ldots, M,
$$

and $\mathbf{G}_{i}$ is the $N$-vector with components

$$
\left(\mathbf{G}_{i}\right)_{k}=h\left(\epsilon_{k}\right) V_{i}\left(\epsilon_{k}\right), \quad k=1, \ldots, N .
$$

We determine the solution $\mathbf{W}_{i}^{\boldsymbol{\lambda}_{i}}$ of the minimum problem by computing the singular value decomposition of the associated Euler equation. In order to fix the optimal $\boldsymbol{\lambda}_{i}$, we adopt the criterion described in [12] and based on an analysis of the cumulative residuals between the input data and the forward-fit values corresponding to the regularized solution. (A similar approach is discussed in [17].)

4.3. Imaging. Once the set $\left\{W_{i}^{\boldsymbol{\lambda}_{i}}\left(E_{j}\right), j=1, \ldots, M\right\}_{i=1}^{L}$ has been obtained, maps of the electron flux spectrum can be reconstructed by applying standard Fourier-based imaging methods. We utilized two algorithms available in SSW:

- Forward-fit $(\mathrm{FF})$. When the source has a relatively simple structure, a fast reconstruction algorithm involves forward-fitting a parameterized geometrical form to the experimental visibilities. SSW utilizes four possible forms of source structure: a circular Gaussian function, a sum of two circular Gaussian functions, an elliptical Gaussian function, and a curved elliptical Gaussian function (mimicking a loop). 
- The maximum entropy method (MEM) [2]. This is an iterative technique searching for the image $F$ maximizing

$$
H=-\sum_{\ell=1}^{n_{\text {pix }}} \frac{F_{\ell}}{F_{T}} \ln \left(\frac{F_{\ell}}{F_{T}}\right),
$$

where $n_{\text {pix }}$ is the number of pixels, $F_{\ell}$ is the content of pixel $\ell$, and $F_{T}=\sum_{\ell=1}^{n_{\text {pix }}} F_{\ell}$. In this method the maximization problem must satisfy two constraints: the total flux $F_{T}$ must be equal to the maximum absolute value of the visibilities, and $\chi^{2}=1$.

In the following two sections, we utilize FF in all tests with synthetic data and MEM when applying the method to RHESSI measurements. For the sake of completeness, we also assessed the behavior of MEM in the case of the synthetic data and found that it performs rather reliably, although with a certain tendency to undersmoothing the shape of the flare and underestimating its size.

5. Testing the method using simulated data. In this section we apply the new imaging spectroscopy method to synthetic data. Construction of the electron maps and comparison with the originally assumed synthetic maps then permit an assessment of the accuracy and fidelity of the method. We consider four sets of simulated data.

5.1. First test: Double footpoints. For the first test case we selected the electron energy range $E=[10,400] \mathrm{keV}$ and divided it into 1950 0.2-keV-wide electron bins. For each electron bin, we defined a theoretical mean source electron flux image representing two separate circular sources ("footpoints"). Each footpoint has a different electron flux spectral index $\delta$, causing the relative brightness of the two sources to vary with energy $E$. The corresponding analytic form is given by

$$
\begin{aligned}
a^{2} N(x, y) \bar{F}(x, y ; E)=\frac{A_{1}}{2 \pi \sigma_{1}^{2}}\left(\frac{E}{40}\right)^{-\delta_{1}} e^{-\frac{\left(x-x_{1}\right)^{2}+\left(y-y_{1}\right)^{2}}{2 \sigma_{1}^{2}}} \\
+\frac{A_{2}}{2 \pi \sigma_{2}^{2}}\left(\frac{E}{40}\right)^{-\delta_{2}} e^{-\frac{\left(x-x_{2}\right)^{2}+\left(y-y_{2}\right)^{2}}{2 \sigma_{2}^{2}}},
\end{aligned}
$$

and we constructed the corresponding $f(\mathbf{x} ; E)$ from (2.5). We utilized the following (arbitrary but plausible) parameters: $\left(x_{1}, y_{1}\right)=(394,454),\left(x_{2}, y_{2}\right)=(404,434)$ (arcsec from solar disk center), $\left(\sigma_{1}, \sigma_{2}\right)=(2,2.5)$ arcsec, $\left(\delta_{1}, \delta_{2}\right)=(4,3)$, and $A_{1}=A_{2}=150$ electrons $\mathrm{cm}^{-2} \mathrm{~s}^{-1} \mathrm{keV}^{-1}$.

We selected the energy range $\epsilon=[10,100] \mathrm{keV}$ and divided it into $N=452$-keV-wide photon bins. For each photon bin, we computed the photon flux $g(\mathbf{x} ; \epsilon)$ emitted from each position $\mathbf{x}$ by numerical integration (Simpson's rule) of the bremsstrahlung equation (2.7), using the cross-section given by formula $3 \mathrm{BN}$ in [8]. We then applied a two-dimensional spatial Fourier transform to the photon maps thus obtained to get the photon visibilities $V_{i}\left(\epsilon_{k}\right)$, $k=1, \ldots, N, i=1, \ldots, L$, at different spatial frequencies. We used 7 subcollimators, with angular resolutions distributed logarithmically from $\sim 7-190$ arcsec, and selected 32 visibilities for each detector, equally distributed azimuthally around the corresponding circle in the $(u, v)$ plane.

Copyright (c) by SIAM. Unauthorized reproduction of this article is prohibited. 


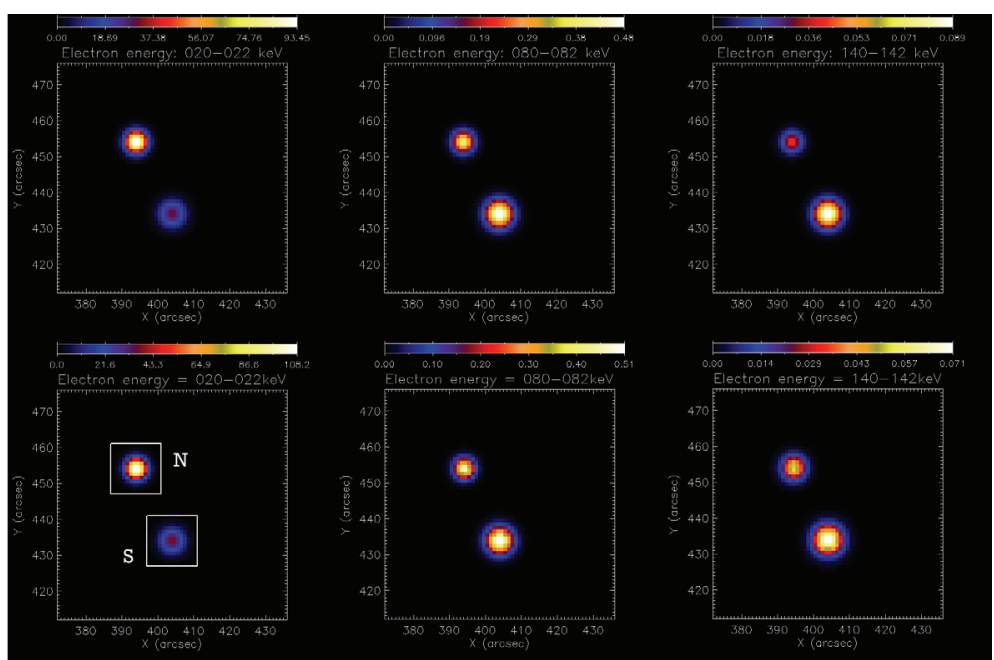

Figure 4. First simulation. Top row: Simulated electron flux maps for 20-22 keV, 80-82 keV, and 140-142 keV energy bins. Bottom row: Recovered electron flux maps using the visibility-based method. The bottom left panel identifies selected subregions of the source.

Then, for each spatial frequency point $\left(u_{i}, v_{i}\right)$, we constructed the photon visibility spectrum $\epsilon_{k} \mapsto V_{i}\left(\epsilon_{k}\right), k=1, \ldots, N$, and applied the Tikhonov regularized spectral inversion method to the discretized version of (3.6). This resulted in a set of $L$ electron visibility spectra $E_{j} \mapsto W_{i}\left(E_{j}\right), j=1, \ldots, M$, with $M=90$. Finally, for each electron energy $E_{j}$, we took all the source visibilities $W_{i}\left(E_{j}\right)$ and applied an SSW FF routine (assuming a double Gaussian source) to construct the electron flux map at energy $E_{j}$. Repeating this for different $E_{j}$ gives the desired imaging spectroscopy result - maps of the source as a function of electron energy $E$. These were then compared to the originally assumed form (5.1) in order to evaluate the accuracy and fidelity of the method.

In Figure 4 we show the theoretical electron maps for the energy ranges 20-22, 80-82, and 140-142 keV (top row; formed by summing the appropriate $0.2-\mathrm{keV}$-bin maps) together with the reconstructed electron images for the same energies (bottom row). Note that both the locations and the relative intensities (which, because the footpoints have different spectral indices, vary with electron energy) of the two bright regions within the source are faithfully recovered. At low (high) energies the more northern (southern) footpoint is brighter.

In order to quantitatively evaluate the accuracy of the reconstructed images, we compared the column-density-weighted electron flux spectra, per unit area within the respective bounding box (electrons $\mathrm{cm}^{-2} \mathrm{~s}^{-1} \mathrm{keV}^{-1} \operatorname{arcsec}^{-2}$ ), extracted from two different spatial subregions around the sources (labelled in the bottom left image of Figure 4, corresponding to the electron energy range 20-22 keV) in the theoretical and reconstructed electron images. These results are shown in panel (a) of Figure 5, where the originally assumed spectra are shown with solid lines and the recovered spectra by a set of points with associated uncertainties shown as error bars. These error bars were calculated as follows:

- Consider a series of realizations of both real and imaginary parts of the photon visibility spectrum. Each realization is produced by adding a randomly assigned noise value, with standard deviation calculated using Poisson statistics.

Copyright ( $)$ by SIAM. Unauthorized reproduction of this article is prohibited. 


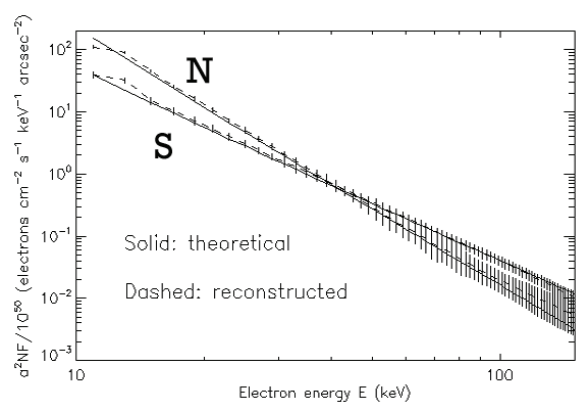

(a)

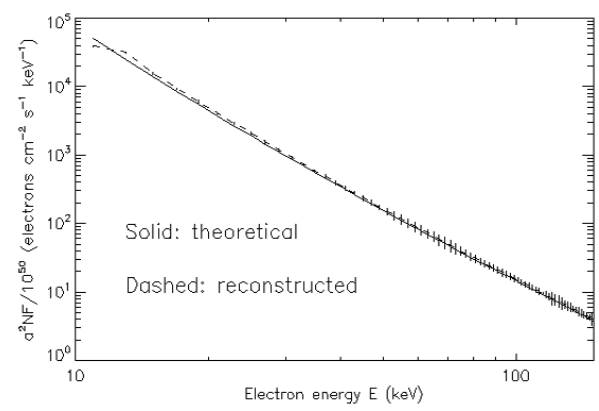

(b)

Figure 5. (a) Original and recovered spectra for the two subregions in the first simulation. (b) Original and recovered spectra for the entire source.

- Repeat the procedure above using the randomized realization of the data.

- Assign to the spatially integrated flux in each designated region or subregion an uncertainty calculated as the standard deviation of the integrated flux values obtained from the different realizations.

Lastly, we calculated the spatially integrated electron flux spectrum in a similar manner, with the results shown in panel (b) of Figure 5.

5.2. Second test: Extended source with spectral dip. For the second test, we constructed a theoretical map consisting of a single curved elliptical Gaussian source, with the electron spectrum constructed from a combination of a "humped" electron spectrum at high energies and a thermal component at low energies. The total spectrum is therefore characterized by a "dip" at intermediate energies. ${ }^{3}$ Specifically, we assumed a spectral form

$$
f_{2}(E)= \begin{cases}100 \cdot e^{-\frac{E}{2.7}}+0.02 \cdot\left(\frac{E}{40}\right)^{7}, & E \leq 40 \mathrm{keV} \\ 0.02 \cdot\left(\frac{40}{E}\right)^{3}, & 40<E<350 \mathrm{keV} \\ 0, & E \geq 350 \mathrm{keV}\end{cases}
$$

and modelled the theoretical mean source electron flux images as

$$
a^{2} N(x, y) \bar{F}(x, y ; E)=C \cdot f_{1}(x, y) \cdot f_{2}(E),
$$

where $C=1.5 \times 10^{7}$ (electrons $\mathrm{cm}^{-2} \mathrm{~s}^{-1} \mathrm{keV}^{-1} \operatorname{arcsec}^{-2}$ ). The intensity and shape of the assumed spectrum correspond to those observed [15] in the July 23, 2002 event, a much more intense event than that considered in the first simulation.

The spatial distribution function $f_{1}(x, y)$ is provided numerically by the appropriate SSW routine, given the coordinates of the source center $[(414,434)$ arcsec], the full width at half maximum of the source $(10 \mathrm{arcsec})$, the eccentricity of the source $(0.8)$, the rotation angle $\left(45^{\circ}\right.$ counterclockwise from the $x$-axis), and the curvature angle of the loop $\left(60^{\circ}\right)$. All other parameters (e.g., energy ranges and bins $(N, M)$ ) are the same as for the double-footpoint test.

\footnotetext{
${ }^{3}$ Note that recovery of such a spectral shape is particularly challenging for forward-fit spectral algorithms [4].
} 


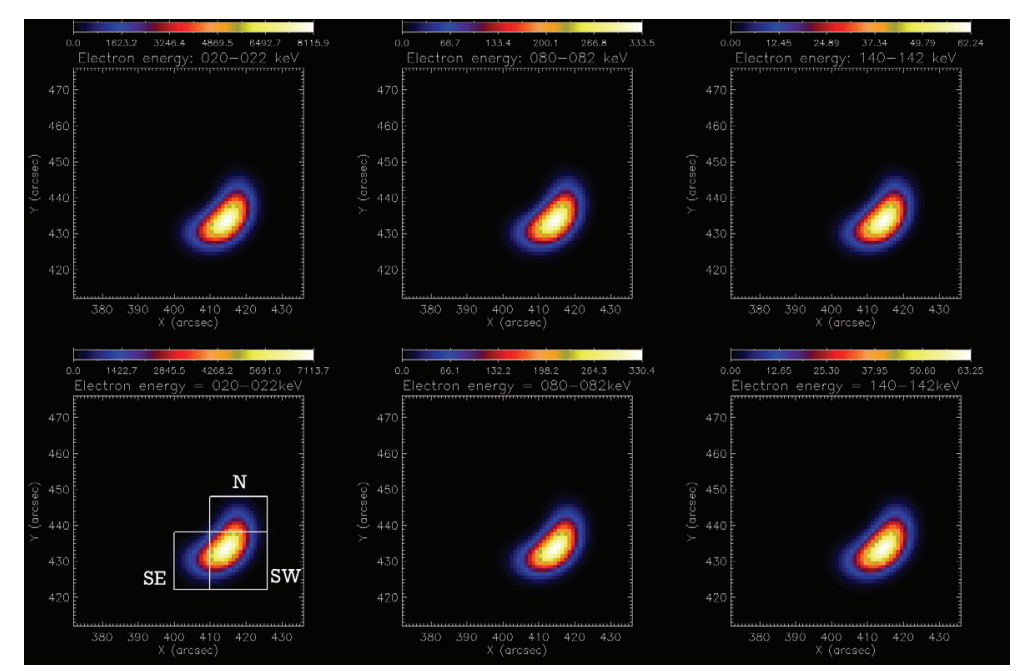

Figure 6. Second simulation. The arrangement of panels is the same as in Figure 4 for the same energy bins, i.e., 20-22 keV, 80-82 keV, and 140-142 keV. Three regions of interest, labelled "N," "SW, "and "SE" (note that west is to the right on the plane of the sky) are indicated in the bottom left panel.

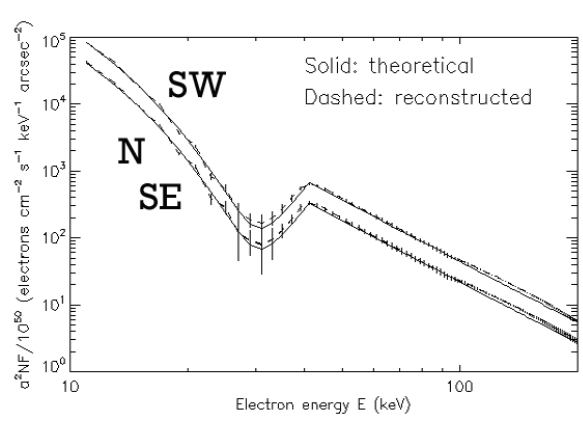

(a)

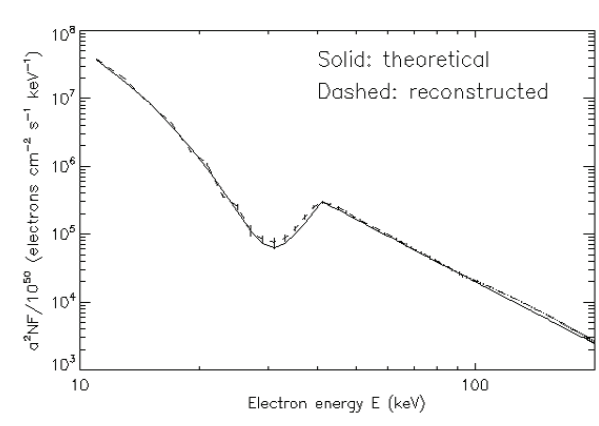

(b)

Figure 7. (a) Original and recovered spectra for the two subregions in the second simulation. (b) Original and recovered spectra for the entire source.

The two rows of Figure 6 are analogous to those in Figure 4. To compare the fluxes, in this case we selected three regions of interest, one around the peak (labelled SW in the bottom left image of Figure 6) and the others near the footpoints (labelled SE and N). In Figure 7 the local and total flux spectra are represented as in Figure 5. The spectra in all three regions are, by construction, identical. This shape (notably the dip at intermediate energies $\sim 30 \mathrm{keV}$ ) is accurately recovered in the high-flux region SW. Even in the low-flux areas SE and N, with their correspondingly poorer statistics, the presence of the dip is still marginally significant.

5.3. Third test: Double footpoints with large brightness ratio. This test deals with the capability of the method to recover weak sources in the presence of brighter ones. We considered again two circular Gaussian sources (see (5.1)) with equal standard deviations $\left(\sigma_{1}=\sigma_{2}=2 \operatorname{arcsec}\right)$ and electron spectral indices $\left(\delta_{1}=\delta_{2}=4\right)$ but with different intensities. We set $A_{1}=150$ (electrons $\mathrm{cm}^{-2} \mathrm{~s}^{-1} \mathrm{keV}^{-1}$ ) and considered different values for $A_{2}$, namely, 


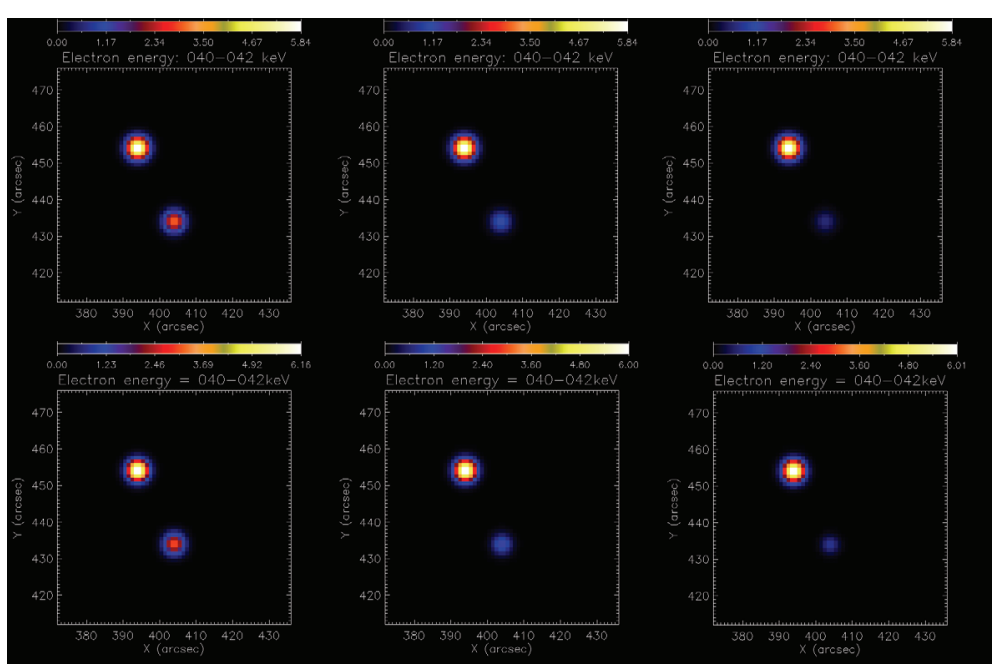

Figure 8. Third simulation. The top row shows the theoretical map for each of three cases; the bottom row shows the recovered maps. The energy bin is the same for all three maps, i.e., 40-42 keV.

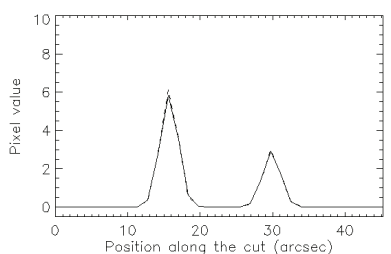

(a)

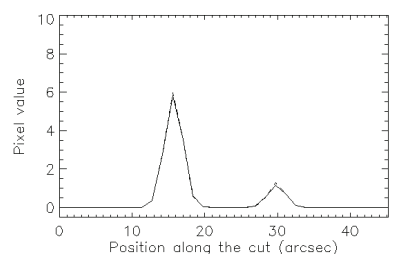

(b)

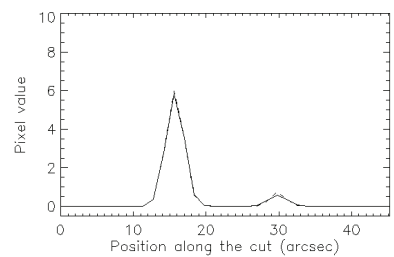

(c)

Figure 9. Intensity along a line connecting the centers of the two sources in the third simulation, with relative brightness of the two sources: (a) $50 \%$; (b) $20 \%$; (c) $10 \%$.

75,30 , and 15 corresponding to $50 \%, 20 \%$, and $10 \%$ of $A_{1}$, respectively. The locations of the two sources, the energy ranges and bins, and the values of $N$ and $M$ are the same as for the first test. In Figure 8 we show the theoretical (top line) and the reconstructed (bottom line) electron maps corresponding to the energy bin 40-42 keV; the first, second, and third columns are for $A_{2}$ equal to $50 \%, 20 \%$, and $10 \%$ of $A_{1}$, respectively. In Figure 9, plots of the theoretical (solid) and reconstructed (dashed) electron flux values along the line connecting the peaks of the two sources are provided. We see that the method faithfully recovers the original source structure, even for a 10:1 ratio in source intensities.

5.4. Fourth test: Double footpoints with energy-dependent size. For this test case we used the double footpoint form (5.1), with $\left(\delta_{1}, \delta_{2}\right)=(5,3)$ and $A_{1}=A_{2}=1000$ electrons $\mathrm{cm}^{-2} \mathrm{~s}^{-1} \mathrm{keV}^{-1}$. The greater difference in spectral indices leads to a larger variation of source intensity with energy. The source size $\sigma_{2}$ was kept constant at 2.5 arcsec, but the source size $\sigma_{1}$ (arcsec) was allowed to vary with energy $E(\mathrm{keV})$ according to the relation

$$
\sigma_{1}(E)=1+\frac{30}{E}
$$

Copyright (c) by SIAM. Unauthorized reproduction of this article is prohibited. 


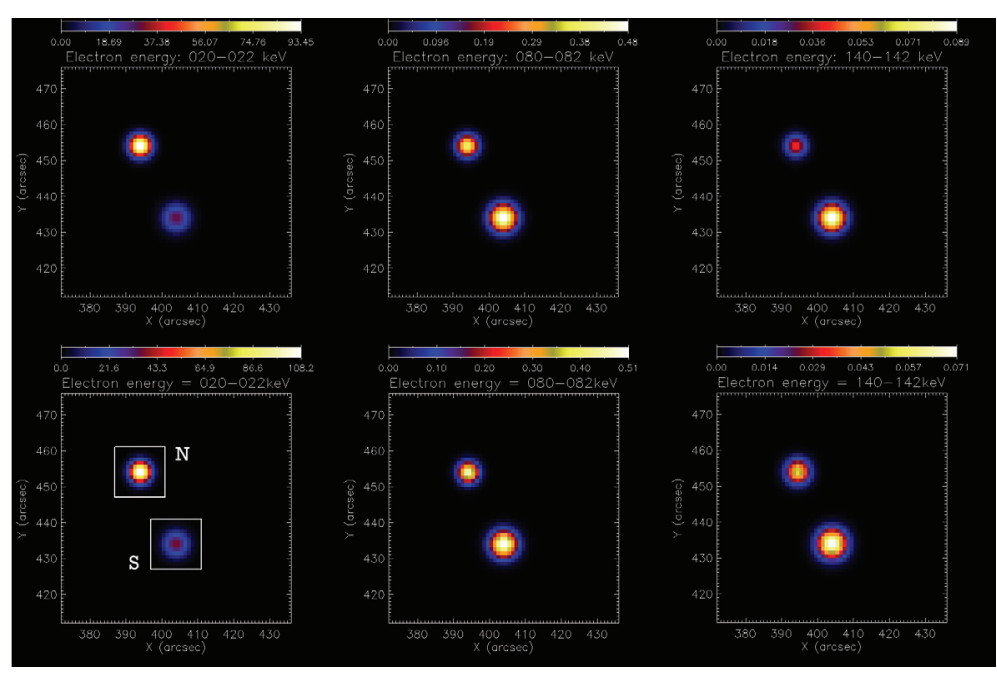

Figure 10. Fourth simulation. Top row: Simulated electron flux maps for 20-22 keV, 80-82 keV, and 140-142 keV energy bins. Note that the size of the top left source decreases with energy (its size is equal to that of the bottom right source at $E=20 \mathrm{keV}$ ). The intensity relative to the bottom right source also decreases with energy; they are equal at $E=40 \mathrm{keV}$. Bottom row: Recovered electron flux maps using the visibility-based method. Two regions of interest, labelled " $N$ " and "S," are indicated in the bottom left panel.

With this choice of $\sigma_{1}(E)$, the two sources are of equal size at $E=20 \mathrm{keV}$; at lower (higher) energies, the first footpoint is larger (smaller), respectively, varying from 4 arcsec at $E=$ $10 \mathrm{keV}$ to $1.2 \operatorname{arcsec}$ at $E=150 \mathrm{keV}$. All other parameters had the same values as in the first simulation.

In Figure 10 we show the theoretical electron maps for the energy ranges 20-22, 80-82, and 140-142 keV (top row), together with the reconstructed electron images for the same energies (bottom row). Note that the locations, relative intensities, and source sizes are all faithfully recovered. It should be noted, however, that this is due to the ability to recover useful visibility values at various locations on the $(u, v)$-plane; in a situation with very low count rates, recovery of useful visibility values may be impossible. As we have verified through simulation, in such a situation the (noisy) visibility data then approaches consistency with a point source at the source centroid.

The areally-averaged, column-density-weighted, electron flux spectra extracted from two different spatial subregions around the sources (labelled in the bottom left image of Figure 10) and for the entire field of view, are shown in panels (a) and (b) of Figure 11, respectively. Here the originally assumed spectra are shown with solid lines and the recovered spectra by a set of points with associated uncertainties shown as error bars.

6. Application to real data. With the viability of the method now demonstrated, we illustrate its use on real data, specifically that obtained during a time interval straddling the X-ray emission peak of the April 15, 2002 (00:11:00-00:12:00 UT) flare. This event has been studied previously by the authors of [20], who used an approach involving forward-fits of photon visibilities.

We used the photon-based visibilities obtained from 7 RHESSI subcollimators spanning 


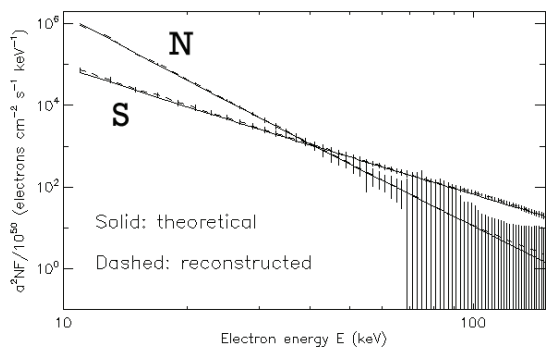

(a)

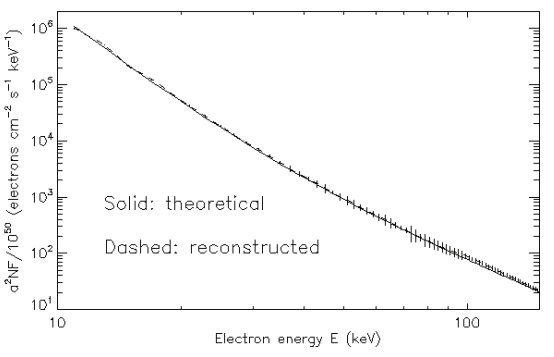

(b)

Figure 11. (a) Original and recovered spectra for the two regions indicated in the bottom left panel of Figure 10. (b) Original and recovered spectra for the entire source.

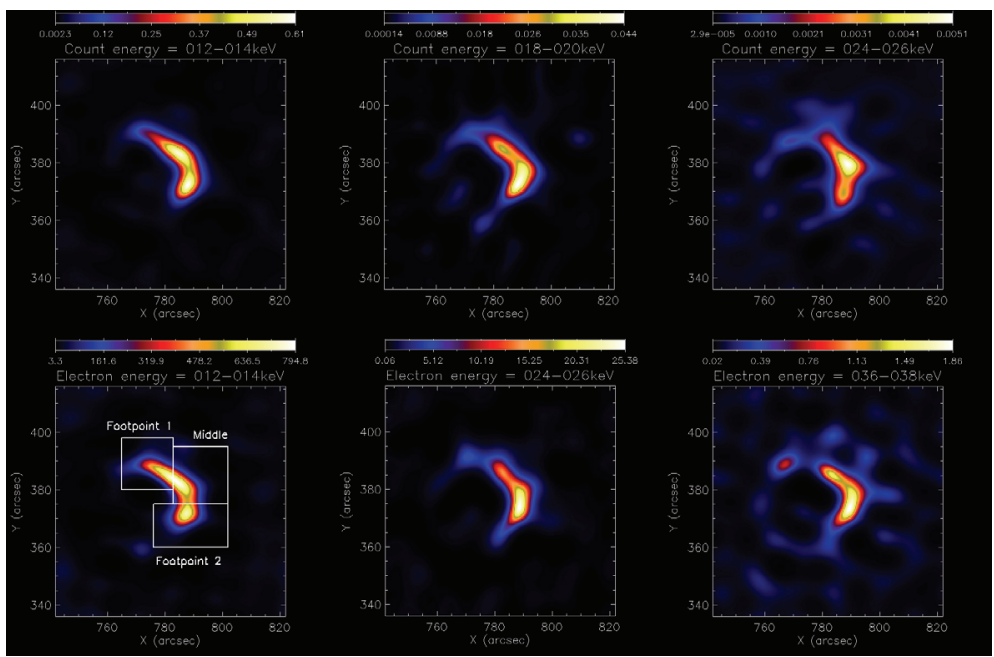

Figure 12. Application of the method to actual data. The energy bins for the 80 arcsec $\times 80$ arcsec photon maps (top row) are (left to right) 12-14 keV, 18-20 keV, and 24-26 keV, respectively. For the electron maps (bottom row), the energy bins are (left to right) 12-14, 24-26, and 36-38 keV. In the bottom row (left panel), we identify three subregions of interest.

spatial resolutions from $\sim 7-190$ arcsec, and applied an MEM imaging algorithm over $N=10$ 2-keV-wide energy intervals from 10-30 keV. In the top row of Figure 12 we show three photon maps corresponding to the energy ranges $12-14,18-20$, and $24-26 \mathrm{keV}$; the pixel resolution is 0.4 arcsec.

Application of our procedure resulted in the production of meaningful electron flux images at energies up to $E \sim 60 \mathrm{keV}$, i.e., $M=25$; a sample of these (corresponding to the electron energy ranges $12-14,24-26$, and $36-38 \mathrm{keV}$ ) are shown in the bottom row of Figure 12. As with the synthetic cases, we considered three different spatial subregions in the source, two of which correspond to the extremities of the source and the other to a location near the center (see the bottom left panel in Figure 12). Figure 13 shows the areally-averaged electron flux spectra for each of the three subregions, and the corresponding (local) spectral indices $\delta=-d \ln F(E) / d \ln E$.

Copyright ( $)$ by SIAM. Unauthorized reproduction of this article is prohibited. 


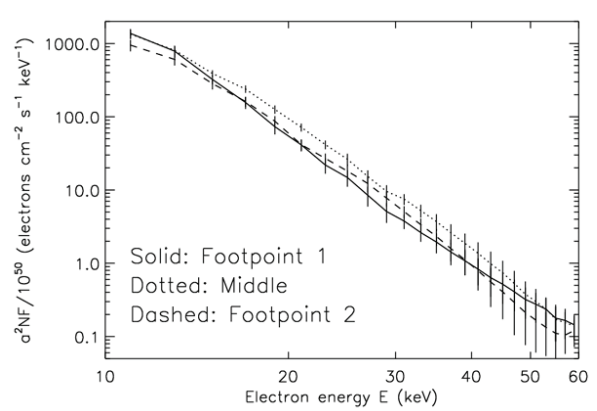

(a)

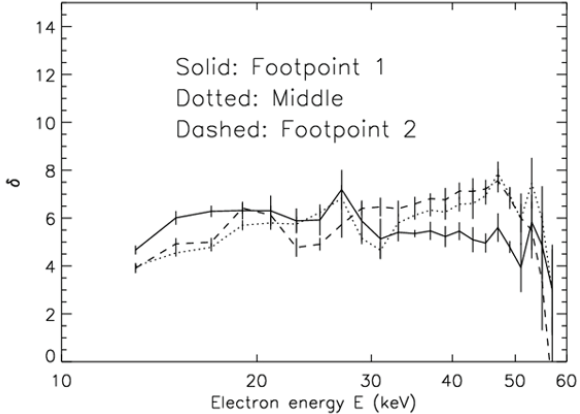

(b)

Figure 13. (a) Electron flux spectra corresponding to the regions highlighted in Figure 12. (b) Corresponding local spectral indices $\delta$.

The spectra for all three subregions of the source are rather similar, as can be inferred from the fact that the shape of the source does not vary substantially with photon (or electron) energy. From $E \sim 30-50 \mathrm{keV}$, the spectral index $\delta$ is slightly smaller (the spectrum is harder) for the "Footpoint 1" subregion near the top of the structure; this is consistent with the appearance of a distinct compact source in the 36-38 keV electron flux image (such a feature is apparent at all electron energies from around $30-60 \mathrm{keV})$. The remainder of the source is quite homogeneous, indicative of either an extended thermal source or an extended acceleration region in a nonthermal source [20]. The near-power-law form of the spectrum in all subregions argues against an isothermal interpretation, although a homogeneous multithermal source (with the same differential emission measure function [16] throughout) is possible. More likely, however, is that the source in Figure 12 represents a dense, extended, acceleration region, as suggested by an earlier photon-visibility-based forward-fit study of the same event [20]. A systematic comparison of the results in [20] with analogous investigations performed by means of our new imaging spectroscopy algorithm is the topic of a new paper currently in preparation.

7. Conclusions. In the present paper we have formulated and validated a new imaging spectroscopy method that uses observed visibilities (here related to photon imaging data) as input and provides as output maps of a source function (in this case the electron flux spectrum) at different values of the source variable (here electron energy). Although we have illustrated the method in the context of RHESSI's RMC-produced data, the approach can also be applied to other observational techniques that use Fourier space information as input data. One such application involves data from a multielement radio interferometer (which also yields Fourier components); however, in such an application the spatial frequencies sampled are themselves frequency-dependent, necessitating that the data be preprocessed using an interpolation/rebinning procedure ("gridding") before direct application of the proposed method.

From a computational viewpoint, an open problem in this approach concerns the effectiveness of the spectral inversion procedure. For example, inversion methods explicitly accounting for the statistical nature of the noise affecting the measured visibilities could be tested. However, it must be noted that the algorithm associated with the Tikhonov method is very rapid, a desirable, if not essential, feature of any useful algorithm. Another open issue concerns 
the imaging algorithm applied to produce the source function maps from the source visibilities: the effectiveness of algorithms (other than FF or MEM) which are capable of exploiting a priori information on the solution is currently under investigation.

\section{REFERENCES}

[1] M. Bertero, Linear inverse and ill-posed problems, in Advances in Electronics and Electron Physics 75, P. W. Hawkes, ed., Academic Press, New York, 1989, pp. 1-120.

[2] S. C. Bong, J. Lee, D. E. Gary, And H. S. Yun, Spatio-spectral maximum entropy method. I. Formulation and test, Astrophys. J., 636 (2006), pp. 1159-1165.

[3] J. C. Brown, Deduction of energy spectra of non-thermal electrons in flares from the observed dynamic spectra of hard X-ray bursts, Solar Phys., 18 (1971), pp. 489-502.

[4] J. C. Brown, A. G. Emslie, G. D. Holman, C. M. Johns-Krull, E. P. Kontar, R. P. Lin, A. M. MAssone, AND M. PiAnA, Evaluation of algorithms for reconstructing electron spectra from their bremsstrahlung hard X-ray spectra, Astrophys. J., 643 (2006), pp. 523-531.

[5] J. C. Brown, A. G. Emslie, And E. P. Kontar, The determination and use of mean electron flux spectra in solar flares, Astrophys. J., 595 (2003), pp. L115-L117.

[6] E. HAug, Photon spectra of electron-electron bremsstrahlung, Solar Phys., 178 (1998), pp. 341-351.

[7] G. J. Hurford, E. J. Schmahl, R. A. Schwartz, A. J. Conway, M. J. Aschwanden, A. Csillaghy, B. R. Dennis, C. Johns-Krull, S. Krucker, R. P. Lin, J. Mctiernan, T. R. Metcalf, J. Sato, And D. M. Smith, The RHESSI imaging concept, Solar Phys., 210 (2002), pp. 61-86.

[8] H. W. Koch And J. W. Motz, Bremsstrahlung cross-section formulas and related data, Rev. Modern Phys., 31 (1959), pp. 920-955.

[9] E. P. Kontar, M. Piana, A. M. Massone, A. G. Emslie, and J. C. Brown, Generalized regularization techniques with constraints for the analysis of solar bremsstrahlung X-ray spectra, Solar Phys., 225 (2004), pp. 293-309.

[10] R. P. Lin ET AL., The Reuven Ramaty high-energy solar spectroscopic imager (RHESSI), Solar Phys., 210 (2002), pp. 3-32.

[11] A. M. Massone, A. G. Emslie, E. P. Kontar, M. Piana, M. Prato, and J. C. Brown, Anisotropic bremsstrahlung emission and the form of regularized electron flux spectra in solar flares, Astrophys. J., 613 (2004), pp. 1233-1240.

[12] A. M. Massone, M. Piana, And M. Prato, Regularized solution of the solar Bremsstrahlung inverse problem: Model dependence and implementation issues, Inverse Probl. Sci. Eng., 16 (2008), pp. 523545.

[13] L. MerTZ, Ancestry of indirect techniques for X-ray imaging, in EUV, X-Ray, and Gamma-Ray Instrumentation for Astronomy and Atomic Physics, Proceedings of the SPIE, Vol. 1159, SPIE, Bellingham, WA, 1989, pp. 14-17.

[14] M. Piana, A. M. Massone, G. J. Hurford, M. Prato, A. G. Emslie, E. P. Kontar, and R. A. SCHWARTZ, Electron flux spectral imaging of solar flares through regularized analysis of hard X-ray source visibilities, Astrophys. J., 665 (2007) pp. 846-855.

[15] M. Piana, A. M. Massone, E. P. Kontar, A. G. Emslie, J. C. Brown, and R. A. Schwartz, Regularized electron flux spectra in the 2002 July 23 solar flare, Astrophys. J., 595 (2003), pp. L127L130.

[16] M. Prato, M. Piana, J. C. Brown, A. G. Emslie, E. P. Kontar, and A. M. Massone, Regularized reconstruction of the differential emission measure from solar flare hard X-ray spectra, Solar Phys., 237 (2006), pp. 61-83.

[17] B. W. Rust And D. P. O'LeARY, Residual periodograms for choosing regularization parameters for ill-posed problems, Inverse Problems, 24 (2008), 034005.

[18] D. M. Sмith et Al., The RHESSI spectrometer, Solar Phys., 210 (2002), pp. 33-60.

[19] A. R. Thompson, J. Moran, And G. Swenson, Interferometry and Synthesis in Radioastronomy, John Wiley, New York, 2001.

[20] Y. Xu, A. G. Emslie, And G. J. Hurford, RHESSI hard X-ray imaging spectroscopy of extended sources and the physical properties of electron acceleration regions in solar flares, Astrophys. J., 673 (2008), pp. 576-585.

Copyright $\odot$ by SIAM. Unauthorized reproduction of this article is prohibited. 